\title{
Entre la ciudad y el campo. Migración por amenidad en la periferia de Buenos Aires
}

Between the City and the Countryside.

Amenity Migration in Buenos Aires' Periphery

Entre a cidade e o campo. Migração devido

a amenidades na periferia de Buenos Aires

María Eugenia Funes*

Recibido: 18 de enero de 2021

Aprobado: 23 de junio de 2021

https://doi.org/10.12804/revistas.urosario.edu.co/territorios/a.10136

Para citar este artículo

Funes, M. E. (2021). Entre la ciudad y el campo. Migración por amenidad en la periferia de Buenos Aires. Territorios, (45), 293-312. https://doi.org/10.12804/revistas.urosario.edu.co/territorios/a.10136

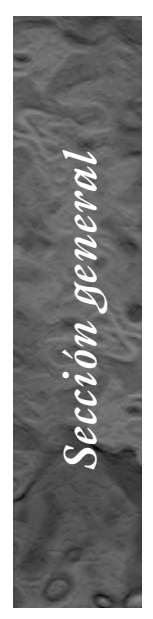

* CONICET con sede en el Programa "Sociedad, Cultura y Religión" del Centro de Estudios e Investigaciones Laborales (CEIL-CONICET). Correo electrónico: maria.funes@usal.edu.ar. ORCID: https://orcid.org/00000002-2980-6396 
Palabras clave

Migración por amenidad; sectores medios; periferias urbanas; Región Metropolitana de Buenos Aires.

Keywords

Amenity migration; middle classes; urban peripheries; Metropolitan Region of Buenos Aires.

Palavras-chave

Migração por amenidades; setores médios; periferias urbanas; Região Metropolitana de Buenos Aires.

territarias 45
RESUMEN

Este artículo tiene como propósito contribuir con la comprensión de los procesos de transformación de las periferias urbanas de Buenos Aires durante las últimas décadas. Para ello se analizará un caso de movilidad residencial hacia una localidad intermedia y periférica de la Región Metropolitana de Buenos Aires por parte de individuos y familias de sectores medios desde la ciudad y desde los centros suburbanos. El artículo se organiza en dos partes. En primer lugar, se identifican las características de este tipo de movilidad residencial en relación con otros desarrollados por estos mismos sectores sociales desde la década de 1980: neorruralismo, migración por amenidad y relocalización en urbanizaciones cerradas. En segundo lugar, se muestra la emergencia de una paradoja particular en las experiencias espaciales de estos nuevos habitantes, quienes experimentan vivir al mismo tiempo en un entorno rural y urbano. Los datos fueron construidos a través de entrevistas en profundidad realizadas en el marco de una investigación etnográfica realizada entre 2014 y 2018.

\section{ABSTRACT}

The purpose of this article is to contribute with the comprehension of the transformations that the urban peripheries during the past decades. With that purpose in mind, I will analyze a case of residential mobility from the city and suburban centers towards an intermediate and peripheric location of Buenos Aires' Metropolitan Region by middle-class individuals and families. The article is organized in two different parts. First, I will identify the characteristics of this spatial process in relation with others developed by the same social sectors since the 1980s: neoruralism, amenity migration and the relocalization in gated communities. Then, I will show the emergence of a particular paradox within the spatial experiences of these new inhabitants who experience living at the same time "in the countryside" and "in the city". The data was collected by means of in-depth interviews in the context of an ethnographic research developed between 2014 and 2018 .

\section{RESUMO}

O objetivo deste artigo é contribuir para a compreensão dos processos de transformação das periferias urbanas nas últimas décadas. Para isso, será analisado um caso de mobilidade residencial para uma localidade intermediária e periférica da Região Metropolitana de Buenos Aires por indivíduos e famílias de setores médios da cidade e de centros suburbanos. O artigo está organizado em duas partes. Em primeiro lugar, são identificadas as características desse tipo de mobilidade residencial em relação a outras, desenvolvidas por esses mesmos setores sociais desde a década de 1980: neorruralismo, migração por amenidades e relocalização em condomínios fechados. Em segundo lugar, é mostrado o surgimento de um paradoxo particular nas experiências espaciais desses novos habitantes, que vivenciam viver ao mesmo tempo em um ambiente rural e urbano. Os dados foram construídos por meio de entrevistas em profundidade, realizadas no âmbito de uma pesquisa etnográfica realizada entre 2014 e 2018. 


\section{Introducción}

Los estudios acerca de las transformaciones que atravesó la periferia de Buenos Aires a partir de la década de 1990 se han concentrado, fundamentalmente, en el análisis de procesos estructurales como la expansión de la mancha urbana, la suburbanización y periurbanización de las localidades de la tercera corona de la $\mathrm{RMBA}^{1}$ (Ciccolella et al., 2015). Estas investigaciones suelen afirmar la transición de un modelo de ciudad compacta (Ciccolella, 2009), formada por una trama urbana continua que implicaba cierta integración entre el centro y sus periferias (Torres, 2009) a una ciudad archipiélago, compuesta por nuevas centralidades en torno a mega urbanizaciones privadas, shopping centers y torres residenciales comunicados por grandes autopistas ( $\mathrm{Cicco}-$ lella, 2009; Gorelik, 2015). Por su parte, el carácter novedoso y disruptivo que tuvo la proliferación de barrios cerrados durante la década de 1990 (Torres, 2001) y, posteriormente, de mega urbanizaciones cerradas (Robert, 2000; Girola, 2004; Ríos \& Pírez, 2008) en la periferia de la ciudad, acaparó la atención de la mayor parte de las investigaciones que analizan las prácticas residenciales de los sectores medios y altos en las periferias de la ciudad. Estos trabajos mostraron la convivencia entre una lógica de profundización de la fragmentación urbana (Torres, 2001; Prévôt -Schapira, 2005) y la dinamización de los mercados de trabajo locales a partir de la instalación de las denominadas nuevas "elites suburbanas" (Pírez, 2009).

A pesar de su importancia para comprender las transformaciones estructurales que atravesaron las periferias de la ciudad durante las últimas décadas, estas investigaciones dejan fuera el estudio de procesos concomitantes, como el que se abordará en este artículo. Analizaré un proceso de transformación de las periferias que aún no ha sido lo suficientemente explorado a nivel local: la movilidad residencial hacia barrios abiertos ubicados en conglomerados intermedios y antiguas áreas de ocio de las periferias urbanas, llevado adelante por individuos y familias de sectores medios. Para ello, analizaré las experiencias espaciales de personas que se trasladaron a la localidad de Ingeniero Maschwitz, ubicada dentro del corredor norte de la periferia de Buenos Aires, a partir de la década de $1990 .^{2}$ Me concentraré en la forma en que los nuevos habitantes de esta localidad explican su llegada a ella, la manera en que viejos y nuevos habitantes interactúan y a las estructuras de sentimientos (Williams, 2011) en torno al espacio que movilizan.

El foco en la forma en que los actores experimentan el espacio se inscribe en una preocupación por reconstruir una imagen compleja de las periferias urbanas en la Argentina (Lacarrieu \& Girola, 2016; Pizarro, 2010; Segura, 2012). Intentaré mostrar que, si bien estas experiencias urbanas muestran tendencias similares a otros procesos de movilidad residencial
${ }^{1}$ El término Región Metropolitana de Buenos Aires hace referencia tanto a la ciudad de Buenos Aires como a sus suburbios $y$ parte de sus periferias. Se compone de a) la Ciudad Autónoma de Buenos Aires, con un área de 200 $\mathrm{km}^{2}$ y una población actual de alrededor de 2,9 millones de habitantes; b) el Conurbano Bonaerense, conformado por 24 partidos que forman la $1^{\circ}$ y $2^{\circ}$ corona de la aglomeración, con un área de $3.627 \mathrm{~km}^{2}$ y una población actual aproximada de 9,9 millones de habitantes; $y$ c) la $3^{a}$ corona, integrada por 16 partidos, con un área aproximada de $15.800 \mathrm{~km}^{2}$ y una población actual de 2,1 millones habitantes. La localidad Ingeniero Maschwitz forma parte de esta última (Ciccolella et al., 2015). La $3^{a}$ corona presentó la mayor variación intercensal de población en el periodo 2001 -2010, pasando de 1.6 a 2.1 millones de habitantes entre 2001 y 2010. Esta variación del $31,3 \%$ supera a la de las primeras dos coronas de la periferia $(13,8 \%)$ y a la de la Ciudad de Buenos Aires (4\%) (INDEC, 2001, 2010).

${ }^{2}$ Como no contamos con datos desagregados por

tersitarios 45

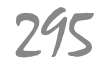


$\Longrightarrow$

localidad, enmarcamoseste crecimiento en el proceso de crecimiento demográfico del partido de Escobar, al cual pertenece Ingeniero Maschwitz. Entre 1991 y 2011 la población de este partido presentó una variación intercensal del 38\% cuando pasó de 128.421 a 178.155 habitantes. En 2010 alcanzó los 213.269 habitantes, con una variación intercensal que disminuyó al 19,7\%, y actualmente se estima que cuenta con 258.805 habitantes (INDEC, 2020) lo cual representaría una variación intercensal del $21,3 \%$. Este crecimiento demográfico no redundó, sin embargo, en una variación de su densidad poblacional media que se mantuvo entre 20 y 1000 babitantes por kilómetro cuadrado (Instituto del Conurbano, 2014a, 2014b).

3 Este artículo es producto del diálogo con una gran cantidad de colegas. Agradezco particularmente a Gabriel Noel, Guillermo Jajamovich, Catalina Monjeau Castro y Taly Barán por sus lecturas atentas y comentarios sin los cuales este trabajo no hubiera sido posible.

4 La antroposofía es una disciplina esotérica creada

territarias 45 296 que llevaron adelante individuos y familias de sectores medios durante las últimas décadas, en esta localidad se produce una paradoja particular: aunque muchas de las personas que llegaron a ella lo hicieron buscando un ritmo de vida "más tranquilo" y un mayor contacto con la naturaleza, esta práctica los vuelve parte de una creciente urbanización que los "invade" unos años después de instalados. ${ }^{3}$

La información fue recolectada a través de una investigación etnográfica (Guber, 2009) realizada en la localidad entre los años 2014 y 2018 durante los cuales participé de distintas actividades junto con estos nuevos habitantes. Conocí Ingeniero Maschwitz en 2014 mientras comenzaba un trabajo de campo sobre la integración entre prácticas espirituales y económicas en los sectores medios de Buenos Aires. Llegué allí cuando me propuse acompañar las actividades de una fundación que promovía el "uso consciente del dinero" y había sido organizada en el marco de las sociabilidades construidas alrededor de una escuela antroposófica de la zona. ${ }^{4}$ El contacto frecuente con las personas que participaban de estos espacios, que habían llegado a la localidad desde principios de la década de 1990, y sus relatos acerca de lo que definían como procesos de "transformación de sus estilos de vida", me llevó a problematizar la dimensión espacial. En esas narrativas el interés y contacto con disciplinas y prácticas espirituales - como la antroposofía, el yoga o la astrología- iba siempre de la mano con una referencia a la "huida de la ciudad" como condición para el desarrollo de un modo de vida más “sano”. Así, los testimonios que ilustran las ideas expuestas en este artículo fueron el resultado de conversaciones informales en el marco de actividades de la vida cotidiana de estos nuevos habitantes, así como de entrevistas no direccionadas. Asimismo, muchas de las descripciones de la localidad son el producto de mi propia circulación a través de ella durante esos años.

\section{Movilidades residenciales de los sectores medios}

La movilidad residencial es un fenómeno multicausal en el que interactúan la dinámica del mercado inmobiliario con las oportunidades, necesidades y expectativas habitacionales de los hogares (Di Virgilio, 2014). Desde la década de 1980 los sectores medios y altos urbanos han llevado adelante distintos procesos de modificación de sus lugares de residencia atravesados por lógicas comunes y particulares al mismo tiempo. En este artículo definimos a los nuevos habitantes de la localidad de Ingeniero Maschwitz como parte de los sectores medios por sus procesos de autoadscripción (Visacovsky, 2018) como por sus consumos y ocupaciones. En términos laborales, son emprendedores, comerciantes y profesionales con ingresos medios y altos, entre los que se destacan arquitectos, ingenieros, economistas que tienen cargos jerárquicos en el ámbito 
corporativo o trabajan de manera autónoma. También encontramos educadores, pequeños productores rurales, comerciantes y artistas.

En primer lugar, varias de esas prácticas residenciales estuvieron movilizadas por lo que Gabriel Noel (2013) llama un "repertorio moral" común de valoración de la naturaleza. Este fue identificado en los imaginarios de quienes trasladaron su residencia permanente a las urbanizaciones privadas de la periferia de Buenos Aires, caracterizados por lo que Florencia Girola (2004) definió como un "imaginario de la pureza" en el que la valoración positiva del contacto con la naturaleza convive con la de la seguridad personal y la voluntad de vivir entre personas con capitales económicos y culturales análogos (Svampa, 2001; Arizaga, 2017). Sin embargo, este repertorio no fue privativo de los procesos de movilidad residencial hacia barrios privados, sino que constituyó uno de los pilares sobre los que se monta otro proceso de "salida de la ciudad" protagonizado por los sectores medios en estas décadas: el de la migración por amenidad, neorruralismo (Trimano, 2019) o como lo definió Julieta Quirós (2019) a partir de una categoría que movilizan los propios actores sociales para describir estos procesos: "irse a vivir al interior".

El concepto de migración por amenidad (Matarrita-Cascante, 2017) o por estilo de vida (Benson \& O' Reilly, 2009) fue creado para definir movimientos poblacionales hacia ámbitos rurales o turísticos motivados por la búsqueda de mayor contacto con la naturaleza, un "ritmo de vida más tranquilo", mejores condiciones climáticas y ambientales, así como la posibilidad de entrar en contacto con culturas diferentes y estilos de vida alternativos. En la Argentina, este fenómeno cobró particular relevancia luego de la crisis económico-social del 2001 e involucró la instalación de individuos y familias de sectores medios urbanos y suburbanos en ciudades intermedias y áreas rurales del país. Distintas investigaciones realizadas en Mar de las Pampas, en la provincia de Buenos Aires (Noel, 2011), el Valle de Conlara, provincia de San Luis (Triví, 2018), y en Traslasierra, provincia de Córdoba (Trimano, 2017; Quirós, 2019) coinciden en la identificación de elementos comunes a todos estos procesos migratorios. En todos estos casos los nuevos habitantes invierten en emprendimientos que contribuyen con el desarrollo del turismo y del mercado de trabajo local (González et al., 2009). Pero, por otro lado, la migración por amenidad da lugar a una "(re)valorización de la tierra que comporta, para buena parte de la población autóctona, efectos de desplazamiento y desposesión” (Quirós, 2019 , p. 278). Además, el contacto entre viejos y nuevos habitantes suele llevar a la formación de identidades contrapuestas (Trimano, 2017) formadas alrededor de controversias sobre problemáticas medioambientales y sobre los usos deseables del espacio (Noel, 2011; Noel \& De Abrantes, 2014).

ner en Alemania en la década de 1910, que llegó a la Argentina a través de migrantes europeos $y$ se compone de diferentes ramas que buscan traducir su cosmovisión con la salud, la medicina, el arte, la religión, la agricultura y la educación (Riera et al., 2018). Como se mostró en otra publicación (Funes, 2021) la localidad de Ingeniero Maschwitz es reconocida en algunos sectores por su circuito de prácticas espirituales (Magnani, 1999; Carozzi, 2000) formado por escuelas antroposóficas, localmente conocidas como "waldorf", comercios especializados en bienes $y$ alimentos para el cuidado bolistico, y consultorios médicos y terapéuticos donde se ofrecen terapias alternativas de todo tipo. Este circuito suele ser indicado como un componente "único" de la localidad que contribuye con el bienestar integral (Viotti, 2017) de sus habitantes.

\section{territarias 45}

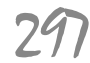


${ }^{5}$ El partido de Escobar fue fundado en 1957 a raiz del crecimiento económico y poblacional de la zona, en un contexto de instalación de industrias y de desarrollo de barrios obreros comunicados de manera deficiente con la ciudad de Buenos Aires (Torres, 1993; Gutman, 2007). Junto con Ingeniero Maschwitz, el partido está formado por otras cuatro localidades y se caracteriza por la convivencia de "áreas que disponen de infraestructura y habitadas por sectores sociales incluidos en sectores laborales de alta calificación, ya sea en los mismos partidos o en otras áreas urbanas del AMBA, junto con áreas marginadas que no disponen de infraestructura urbana y que se insertan esporádicamente en sectores laborales de baja calificación, en actividades de servicios, ma nufactura o agropecuarias tanto locales como en otras áreas del AMBA" (Pizarro, 2010, p. 94).

\section{territarias 45} 298
Por otra parte, durante las últimas décadas los sectores medios desarrollan procesos de movilidad residencial motivados por ganancias de localización (Bourdieu, 2000) prácticamente opuestas a las anteriormente mencionadas, como la búsqueda de centralidad. A diferencia de otras ciudades latinoamericanas, la ciudad de Buenos Aires presenta un tipo de movilidad centrípeta que incluye a individuos y familias que se mudan a barrios céntricos de la ciudad desde sus suburbios y desde áreas urbanas y rurales de otras provincias. En estos casos prevalece la búsqueda de cercanía con los lugares de trabajo, con mejores sistemas de transporte público, así como con una amplia oferta de servicios urbanos y comerciales (Cosacov, 2014). Esta búsqueda de centralidad fue también identificada en los procesos de densificación y verticalización de los subcentros de los suburbios de Buenos Aires (Ciccolella et al., 2015) y en los procesos de gentrificación y de elitización de los centros de ciudades intermedias de la Patagonia (Perren \& Cabezas, 2018). Asimismo, Mercedes Di Virgilio (2014) mostró que, al menos la mitad de las prácticas de movilidad residencial en el Área Metropolitana de Buenos Aires están influidas por la búsqueda de proximidad respecto de la vivienda de origen, y Natalia Cosacov (2017) dio cuenta del papel central que las redes familiares tienen en las trayectorias residenciales y la movilidad cotidiana de una parte de los sectores medios de Buenos Aires.
A continuación, se analizarán las características de la movilidad residencial hacia la localidad de Ingeniero Maschwitz a la luz de las características de los procesos de movilidad residencial llevados adelante por individuos y hogares de sectores medios para encontrar sus elementos comunes y particularidades.

\section{La suburbanización de Ingeniero Maschwitz}

Ubicada a $44 \mathrm{~km}$ al noreste de la ciudad de Buenos Aires, en el límite actual entre la mancha urbana y las áreas predominantemente rurales de la provincia de Buenos Aires, Ingeniero Maschwitz es una de las cinco localidades que conforman el partido de Escobar. ${ }^{5}$ Desde sus inicios, esta localidad presentó una composición territorial heterogénea: allí convivieron estancias, pequeñas unidades agrícolas, áreas comerciales, sectores residenciales $\mathrm{y}$, conforme nos acercamos al final del siglo $\mathrm{Xx}$, barrios de casas quintas y urbanizaciones privadas. Esta heterogeneidad, así como su locación entre las mega urbanizaciones cerradas que se extienden en los partidos linderos de Tigre y Pilar (Ríos \& Pírez, 2008; Lacarrieu \& Girola, 2016) y áreas rurales (Pizarro, 2010) representan un desafío analítico que convierte a la localidad en un territorio difícil de definir por medio de las tipologías urbanas disponibles.

El pueblo de Ingeniero Maschwitz fue fundado en 1910 en una zona de grandes 
propiedades agropecuarias pertenecientes a familias de elite que residían en la ciudad de Buenos Aires. Su creación estuvo ligada a la construcción de una estación de ferrocarril (figura 1) que la comunica con la ciudad de Buenos Aires ${ }^{6}$ y el loteo de parte de una estancia para la comercialización de terrenos destinados a la construcción de viviendas y comercios. Allí se instalaron familias de migrantes europeos que arrendaban tierras en las que desarrollaban actividades agropecuarias y hortícolas, que caracterizaron la economía de la localidad durante la mayor parte del siglo Xx (Bozzano, 2007).

Figura 1. Estación de ferrocarril de Ingeniero Maschwitz

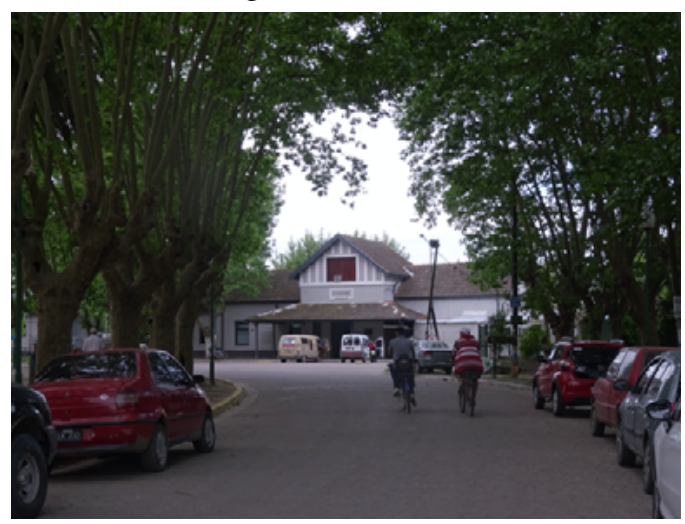

Fuente: archivo personal.

Esta configuración territorial se vio modificada con la llegada de familias de sectores medios y altos provenientes de la ciudad de Buenos Aires y de localidades consolidadas de los suburbios. Este proceso se produjo en dos etapas caracterizadas por patrones residenciales diferentes. En primer lugar, a partir de la década de 1960, se lotearon terrenos de viejas estancias para la creación de dos barrios de casas de fin de semana y tres clubes deportivos. Estos limitaban con otros barrios similares ubicados en localidades vecinas, como Benavidez o Pilar. Así, una parte de Ingeniero Maschwitz se integró con una serie de barrios de amplios terrenos que contaban con solo algunos servicios, como electricidad y alumbrado público. El desarrollo de este tipo de barrios se explica, en parte, por la expansión del automóvil entre los consumos de los sectores medios urbanos, la modernización de las infraestructuras viales, en este caso la ruta Panamericana (Gutiérrez, 2007; Gómez Pintus, 2013), y la ampliación de los espacios de ocio y deporte utilizados por las elites y clases medias de Buenos Aires (Ballent, 1998; Robert, 2000; Ballent y Gorelik, 2001; Gorelik, 2015). Esta primera oleada de individuos que utilizaban la localidad como residencia temporal durante fines de semana y vacaciones no afectó significativamente la vida cotidiana de los habitantes de la localidad.

Luego, a partir de la década de 1990 se produjo una nueva etapa de transformaciones con la construcción de cinco barrios cerrados, un creciente uso de las casas de fin de semana existentes como residencias permanentes (figuras 2 y 3 ) y la comercialización de lotes para la construcción de viviendas en áreas hasta
${ }^{6}$ En la actualidad, este viaje lleva aproximadamente una hora y media, implica una combinación de trenes en Villa Ballester y se puede hacer una vez cada dos horas. 
${ }^{7}$ Los barrios y localidades tipicamente asociados a este corredor son los barrios de Recoleta, $\mathrm{Pa}$ lermo y Belgrano, en la ciudad de Buenos Aires; y las localidades de Vicente López, Olivos y San Isidro, en el sector norte de los suburbios.

\section{territarias 45} 300 entonces rurales. Este crecimiento fue, nuevamente, habilitado por la ampliación y la mejora de la autopista Panamericana (Torres, 2001). En estas nuevas áreas residenciales se instalaron individuos y familias, la mayoría provenientes de lo que suele denominarse el "corredor norte", conformado por barrios de la ciudad de Buenos Aires y de los suburbios con una gran concentración de habitantes de sectores medios y altos ${ }^{7}$ (Di Virgilio, 2014).

Esta característica, junto al hecho de que estos barrios están comunicados entre sí por una autopista por la que circulan numerosas líneas de transportes colectivos públicos, implica que muchos de los nuevos habitantes de Ingeniero Maschwitz pueden mantener un contacto frecuente con sus barrios de origen, donde suelen vivir familiares y amigos. Por otra parte, algunos de estos nuevos habitantes utilizan la localidad como un suburbio, ya que continúan viajando a la ciudad durante la semana para trabajar. Otros, en cambio, generaron emprendimientos laborales en la localidad, trabajan en sus casas o en áreas de oficinas e industrias en localidades cercanas.

El crecimiento poblacional de la localidad y de sus zonas aledañas trajo importantes consecuencias económicas entre las que se destacan el aumento del precio de la tierra, la generación de puestos de trabajo y la transformación y multiplicación de las áreas comerciales. El antiguo centro comercial, que se extiende alrededor de la plaza principal, creció con la proliferación
Figuras 2 y 3. Barrios de casas de fin de semana
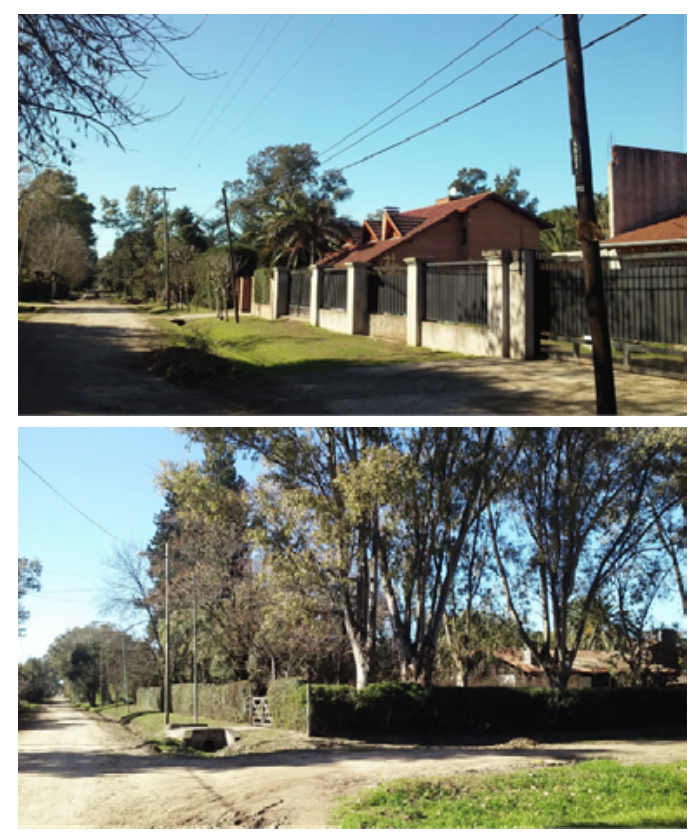

Fuente: archivo personal.

de emprendimientos comerciales y la instalación de sucursales de bancos, grandes cadenas de supermercados y franquicias gastronómicas. Asimismo, los nuevos habitantes e inversores que no viven en la localidad construyeron locales comerciales en distintos puntos de la localidad (figura 4). Uno de los más llamativos es un circuito gastronómico y comercial sobre una calle antiguamente poblada por casas de fin de semana, que presenta varias continuidades con otros desarrollos análogos en la ciudad de Buenos Aires (González Bracco \& Arizaga, 2019). Allí abrieron restaurantes y galerías comerciales con 
diseños estéticamente llamativos que, en algunos casos, integran elementos sustentables. Una de estas galerías, que suele ser definida como vintage, está construida en chapas y materiales reciclados pintados con pátinas de distintos colores. Otra está formada por edificios de formas irregulares que rodean árboles añejos hechos de madera y chapas. Una última está construida con contenedores reciclados e incluye techos vivos. ${ }^{8}$ Así, durante los últimos años, la localidad comenzó a ser presentada en medios de comunicación y redes sociales de viajes como un lugar que "está de moda", con una amplia oferta gastronómica, artística y de diseño, así como un destino de fin de semana cerca de la ciudad a donde es posible ir a "desconectar", encontrar alimentos orgánicos y pasar el día en el aire libre.

\section{Mudarse a la periferia: entre el neorruralismo y la suburbanización}

Los motivos por los cuales los nuevos habitantes de Ingeniero Maschwitz explican su llegada a la localidad presentan varias continuidades con otros procesos de movilidad de los sectores medios y elites. Como se indicó en la introducción, estos suelen presentar una estructura de sentimientos (Williams, 2011) de rechazo a la ciudad, ${ }^{9}$ a la que asocian con un modo de vida nocivo que se contrapone con la búsqueda de un mayor "contacto con la naturaleza". ${ }^{10}$ Estos motivos conviven con
Figura 4. Venta de casas de fin de semana para la construcción de paseos comerciales

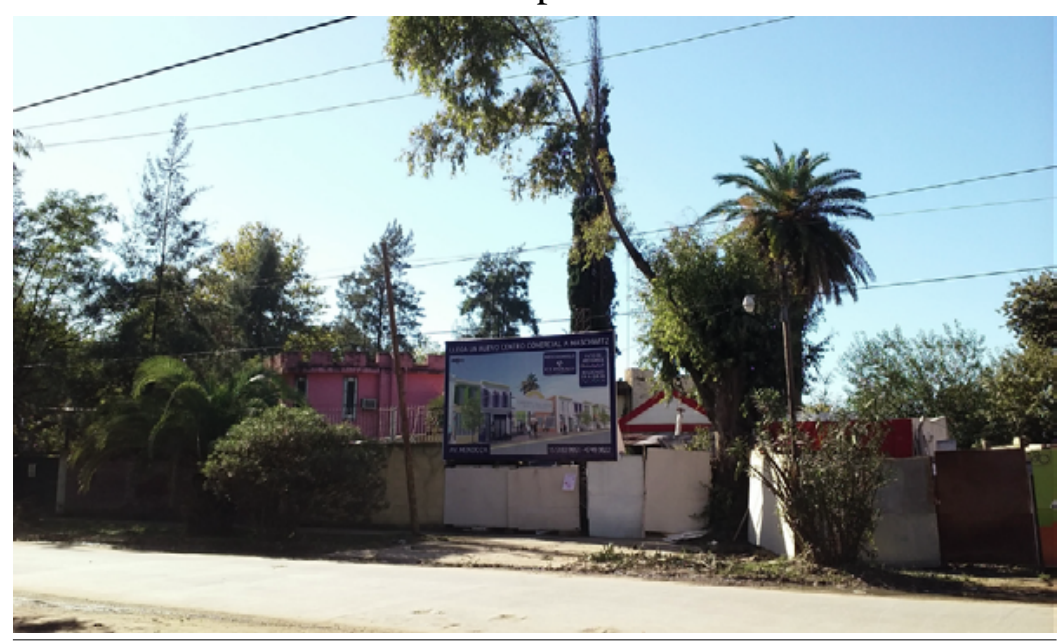

Fuente: archivo personal.

otros materiales, como la posibilidad de acceder a grandes terrenos (de $25 \mathrm{~m}$ por $50 \mathrm{~m}$ y de $50 \mathrm{~m}$ por $50 \mathrm{~m}$ ) con amplios parques por un precio equivalente al de propiedades mucho menores, como un departamento de dos ambientes en barrios más consolidados.

Por otro lado, al igual que en otros procesos de migración por amenidad, la movilidad residencial hacia esta localidad periférica dio lugar al contacto entre sectores sociales con diferentes modos de vida, capitales educativos y económicos. Ambos sectores entran frecuentemente en contacto en el marco de relaciones de intercambio específicas: los nuevos habitantes consumen en negocios manejados por viejos habitantes, muchos de estos últimos trabajan para los primeros, ofreciendo tareas de cuidado y servicios en sus hogares
${ }^{8}$ Los techos "pivos" o verdes son construcciones que se colocan sobre los techos y que permiten que crezcan plantas sobre ellas. Suelen ser utilizados con un fin ecológico, dado que funcionan como aislantes térmicos y contribuyen con la disminución del dióxido de carbono en el aire.

9 Siguiendo a Williams (2011) las categorias de ciudad y campo ban sido movilizadas para oponery contrastar modos de vida opuestos y contrastantes. Las caracteristicas subjetivas con las que cada tipo de asentamiento fue asociado y las estructuras

tersitarios 45 301 
de sentimiento que generaron han variado a lo largo de la historia. Así, en distintos momentos históricos el "campo" ha sido asociado a la paz, la inocencia y la virtud; $y$ en otros al atraso, la ignorancia y los limites al desarrollo humano. Asimismo, la ciudad se ha asociado tanto con el progreso, las comunicaciones, la ilustración como con la alienación, el ruido y la ambición.

${ }^{10}$ Para un análisis acerca de la forma en que los usos $y$ apelaciones a la naturaleza pueden operar como recursos de legitimación $y$ mecanismos de ocultamiento de los procesos de segregación urbana ver Carman (2011) de Buenos Aires.

\section{territarias 45} 302 y emprendimientos económicos. Pero, en general, a mbos sectores no forman lazos de sociabilidad. Ello se explica, en parte, por el hecho de que muchas de sus actividades cotidianas (fundamentalmente, las educativas y de ocio) tienen lugar en espacios e instituciones diferentes.

Asimismo, las diferencias en los modos de vida y los capitales de nuevos y viejos habitantes, suelen dar lugar a la construcción de identidades opuestas. En sintonía con la imagen negativa que traen de "la ciudad", los recién llegados suelen caracterizar a los viejos habitantes, a quienes se refieren como "la gente del pueblo", como portadores de características como la "sencillez", la "tranquilidad" y la "solidaridad". La categoría de "pueblo" suele ser también movilizada por los nuevos habitantes para hacer referencia a un sector particular del espacio: el área formada por el antiguo centro comercial y político-administrativo de la localidad y por un conjunto de manzanas de uso residencial que coincide con el primer trazado de la localidad. Por otra parte, algunos reconocen que muchos de los habitantes "del pueblo" presentan sentimientos de hostilidad hacia ellos por haberlos "invadido". En ese sentido, una maestra de 45 años que se mudó a Ingeniero Maschwitz en 2007 para trabajar en una de las escuelas antroposóficas de la zona me contaría sobre su proyecto de realizar talleres orientados a "la gente del pueblo" para compensar el impacto que generaba la llegada de gente como ella a este lugar:
Yo ahora tengo ganas de hacer algunos talleres. Para ver si la escuela puede dar algo a este barrio que ya es un caos. (...) Yo siempre digo: nosotros vinimos a buscar esta tranquilidad, pero trayendo por otro lado demasiado ruido, tenemos que recordar qué vinimos a buscar ¿no? Entonces buscar un equilibrio entre el crecimiento... porque vinimos a buscar esta paz, vinimos a buscar el barro, entonces, de repente, ahora asfaltar todo. (Comunicación personal, 21 de julio de 2016)

Por su parte, los viejos habitantes suelen remarcar el crecimiento urbano como un fenómeno positivo desde el punto de vista de la dinamización del mercado de trabajo, pero, al mismo tiempo, lamentan los efectos que, la proliferación de autopistas, barrios cerrados y áreas comerciales, tuvo sobre su modo de vida y sobre el medio ambiente. En ese sentido, en una ocasión, mientras se quejaba por los problemas de basura que estaba trayendo "la gente de los countries", una mujer de 70 años que vivió toda su vida con su familia en una quinta de media manzana $(50 \mathrm{~m}$ por $50 \mathrm{~m} \mathrm{)}$, que linda con un viejo club de campo me dijo: "No sabes lo lindo que era esto cuando nos mudamos acá, era un bosque, ahora talaron todo. Nos conocíamos todos, hasta a los ladrones los conocíamos" (Comunicación personal, 9 de marzo de 2017).

Así, nuevos y viejos habitantes se distinguen a partir de su tiempo de residencia en la localidad y por los diferentes 
modos de vida que los caracterizan. En general, esta diferenciación se materializa en la localización de sus residencias, prácticas cotidianas y de ocio, así como muchos de sus trayectos cotidianos. Las desigualdades estructurales de ambos sectores se articulan, entonces, con una distribución desigual y trayectorias de circulación diferentes a lo largo del espacio (Bourdieu, 2000; Heredia \& Di Virgilio, 2012). Por otra parte, esta segregación entre distintos sectores sociales presenta algunas particularidades en relación con procesos similares en otros sectores de las periferias urbanas ( Pírez, 2009; Segura, 2012). Si bien algunas de las zonas residenciales en las que se instalaron individuos y familias de sectores medios están separadas del resto de la localidad por la autopista Panamericana, por cursos de agua o por el tendido del ferrocarril no encontramos aquí ni los paredones ni las medidas de seguridad que separan a quienes viven en barrios cerrados del resto de la ciudad. La articulación entre territorio, diferencia y desigualdad (Carmán et al., 2013) tiene lugar, entonces, a través de la residencia, la formación de redes de sociabilidad y la construcción de identidades opuestas.

Hasta aquí, se enumeraron algunos elementos que la movilidad residencial hacia las periferias presenta respecto de otros procesos de movilidad residencial desarrollados por los sectores medios y altos durante las últimas décadas: la búsqueda de mayor contacto con la naturaleza y la generación de tensiones y de identidades contrarias entre viejos y nuevos habitantes. A continuación, analizaremos una de las particularidades que presentan los migrantes por amenidad que llegaron a Ingeniero Maschwitz que los distinguen de esos otros procesos de movilidad.

\section{El campo en la ciudad}

En muchos sentidos, quienes se trasladaron hacia barrios creados para residencias de fin de semana consideran que viven "como en el campo". Estos barrios carecen de varios servicios, como el agua potable y el saneamiento que deben ser gestionados por cada hogar. Además, sus calles tienen un alumbrado público deficiente y la mayoría son de barrio y no cuentan ni con veredas ni alcantarillados. Esta carencia implica que la llegada de fuertes lluvias suele provocar una serie de dificultades para transportarse, que se vuelve automáticamente el centro de las conversaciones entre los nuevos vecinos. Este carácter extraordinario se debe, probablemente, al hecho de haber crecido en barrios más urbanizados donde no se enfrentaban con algunas de las dificultades que involucra vivir, como muchos lo denominan, "como en el campo".

Una de esas dificultades, tal vez la principal, es el barro (figura 5). Las referencias al barrio son centrales en las experiencias urbanas de los nuevos habitantes de la localidad. Ello se observa, por ejemplo, en la recurrente mención y territarias 45

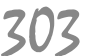


consejo en torno a modelos, cantidades y calidades de botas de lluvia, objetos que son además obsequiados e intercambiados con frecuencia. Por su parte, las anécdotas de haber tenido que dormir en casas de amigos y familiares luego de la sorpresiva llegada de una tormenta no resultan extrañas y cualquier persona tiene un par de botas de lluvia extra a mano para prestar en caso de que un amigo o amiga esté de visita y tenga que salir a la calle cuando todo está embarrado. $\mathrm{Y}$, como pude comprobar en varias ocasiones en que me dirigía caminando a realizar alguna actividad con zapatillas, patinando y esquivando grandes charcos de agua, son necesarias. "No podés vivir en Maschwitz si no tenés botas de lluvia" es una frase que escuché en varias ocasiones y que resume los límites materiales que representa vivir en una zona baja, húmeda y en barrios en los que la mayoría de las calles son de barro o, en el mejor de los casos, de arena.

Pero el barro no es solo evocado para hacer referencia a las dificultades que los nuevos habitantes enfrentan en este tipo de urbanizaciones, sino que es también movilizado como un indicador de rusticidad, contacto con la naturaleza y de haber abandonado una vida nociva en la ciudad. De acuerdo con estos migrantes por amenidad, su experiencia de vivir "como en el campo" se expresa también en el desarrollo de relaciones de reciprocidad e interacciones diferentes a las que presentaban los vínculos entre vecinos en sus barrios de origen. Muchos de mis
Figura 5. Camino embarrado luego de una lluvia en un barrio residencial

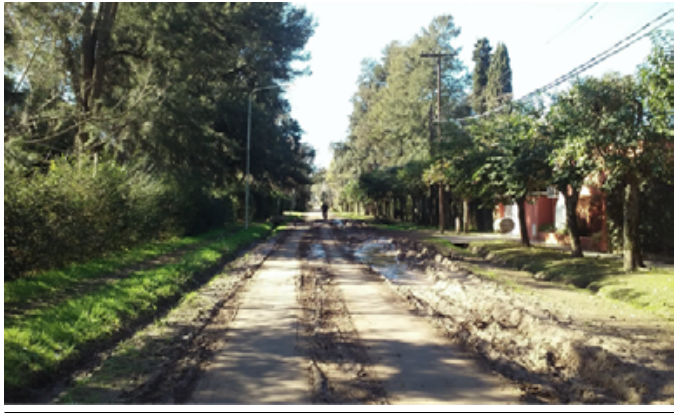

Fuente: archivo personal.

interlocutores definían estos lazos como comunitarios y solidarios. De hecho, durante mi trabajo de campo fui testigo de prácticas de reciprocidad que, en tanto habitante de una gran ciudad, me resultaron extrañas. Durante uno de mis intercambios con una informante le pregunté, por ejemplo, cómo hacían sus hijos para recorrer las casi 20 cuadras sin veredas que separan su casa de la autopista, donde toman diariamente el colectivo que los lleva a la universidad en la ciudad de Buenos Aires. Su respuesta, que escuché con cierto escepticismo, fue que muchas veces alguien "los levantaba" con el auto mientras caminaban al borde de la calle. Mi escepticismo fue puesto a prueba en numerosas ocasiones cuando personas desconocidas frenaron a mi lado mientras caminaba y se ofrecieron a "acercarme" algunas cuadras para que no caminara bajo el sol o para que pudiera llegar más rápido a mi destino. 
Esta mayor cercanía y contacto con los vecinos puede también provocar rechazos entre quienes llegan "desde la ciudad". Durante mi trabajo de campo algunos de mis interlocutores se quejaban de la imposibilidad de "pasar desapercibidos" y añoraban el carácter anónimo que tenían muchas de sus interacciones con vecinos cuando vivían en la ciudad (Wirth, 1945). En ese sentido, un psicólogo y monje budista de 50 años, que se mudó desde la localidad vecina de Tigre en 2011 se sinceró:

[Ingeniero Maschwitz] es un pueblo, y como todo pueblo chico, y esto yo lo aprendí a fuerza de sudor y sangre, tiene sus cosas de pueblo chico infierno grande. Yo en Tigre no estaba acostumbrado a esto porque Tigre es una gran ciudad ya. Y tiene otro espíritu, es como que cada uno está más en la suya. (Comunicación personal, 21 de abril de 2017)

Estas experiencias de "vivir como en el campo" se contraponen con otras que muestran el carácter crecientemente "urbano" de la localidad. Una de las mayores expresiones de esta "cementización", como la denominó una de mis interlocutoras frecuentes, tiene lugar en el tránsito. El automóvil suele ser señalado como un objeto típico de la experiencia espacial en esta localidad y como símbolo de un crecimiento que suele ser caracterizado como excesivo y desordenado. La mayor cantidad de automóviles (figura 6) se vincula no solo con el crecimiento demográfico y la llegada de individuos y familias de sectores medios, sino también con la falta de transportes públicos que comuniquen puntos de la localidad entre sí. Si bien la localidad está conectada con otros centros urbanos a través de avenidas y autopistas, los trayectos cotidianos dentro de ella requieren de un transporte propio, como una bicicleta o un automóvil, propio y contratado a través de agencias o aplicaciones. El automóvil es el principal medio de transporte "posible" para muchos de los nuevos habitantes de la localidad, a tal punto que, varias personas que conocí a lo largo de mi trabajo de campo no utilizaban - y en muchos casos ni siquiera conocían - los recorridos de las líneas locales de colectivos públicos y los horarios del ferrocarril.

Figura 6. Autos estacionados en una de las avenidas principales de la localidad

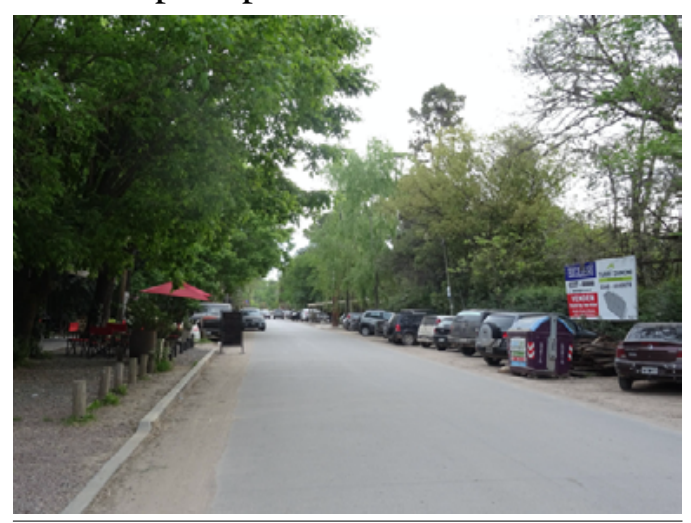

Fuente: archivo personal. territarios 45 305 
Además, como muchos barrios están delimitados por cursos de agua superficial o por el tendido del ferrocarril, con muy pocos puentes que los comuniquen con su entorno, y como la autopista suele congestionarse, moverse hacia otras áreas dentro de la localidad o barrios limítrofes puede involucrar una cantidad considerable de tiempo. Esta mayor cantidad de automóviles que circulan por la localidad implicó el aumento de la contaminación sonora en las áreas comerciales y la emergencia por embotellamientos en locaciones específicas, como las intersecciones entre la autopista Panamericana y otras calles y avenidas. El frecuente uso del automóvil es experimentado por muchos de sus nuevos habitantes de la localidad como una contradicción frente a la búsqueda de "contacto con lo verde" que los atrajo de la zona. En ese sentido, Gonzalo, un arquitecto de 30 años que llegó en 1993 junto con sus padres a una casa de fin de semana por el trabajo de su papá, indicaba:

Lo que es un bajón de Maschwitz es que es un lugar de auto, y cada vez más, es una locura. Esta calle a donde doblás, antes de la Panamericana llegabas al toque, ahora tenés una cuadra de cola, a cualquier hora del día. Todas las familias tienen mínimo dos autos. En algún momento va a ser una locura vivir en Maschwitz, ya se putean en la calle. (Comunicación personal, 19 de territarias 45 mayo de 2017)
La experiencia espacial de los nuevos habitantes de Ingeniero Maschwitz se caracteriza, entonces, por la paradoja de experimentar vivir en el "campo" y en la "ciudad" al mismo tiempo. A pesar de que varios de estos migrantes por amenidad llegaron allí buscando alejarse de los problemas típicos de la ciudad, algunas de sus experiencias espaciales, como la circulación a través de la localidad, pueden involucrar algunos de los problemas "urbanos" de los que escaparon. De hecho, varios de estos problemas han llevado a la organización de protestas y la recolección de firmas para reclamar a las autoridades estatales por obras de prevención de inundaciones, por el establecimiento de límites al crecimiento de áreas comerciales y por la protección de una reserva ecológica situada en la localidad. De esta forma, la vida cotidiana en esta localidad constituye un caso más que problematiza la dicotomía urbano-rural, que en muchas ocasiones continúa guiando la forma en que pensamos el espacio (Noel, 2017).

\section{A modo de conclusión}

En este artículo se analizó el proceso de movilidad residencial llevado adelante por individuos y familias de sectores medios hacia las periferias de Buenos Aires, a partir del caso de la localidad de Ingeniero Maschwitz. Caracterizada por la presencia de clubes de campo y barrios antiguamente destinados a viviendas de 
fin de semana, Ingeniero Maschwitz comenzó a atraer a individuos y familias de sectores medios y altos, en la década de 1960, que utilizaban la localidad como lugar de residencia temporal. A partir de la década de 1990 comenzó a recibir a migrantes por amenidad que se trasladaron allí para, en sus propios términos, alejarse de un modo de vida "urbano", estar en mayor contacto con la naturaleza $\mathrm{y}$ acceder a grandes terrenos.

Este proceso de movilidad presenta algunas continuidades con otros procesos de movilidad residencial protagonizados por otros sectores sociales durante las últimas décadas. Al igual que en el neorruralismo, la migración por amenidad y la relocalización en urbanizaciones cerradas, los nuevos habitantes llegaron a Ingeniero Maschwitz en búsqueda de un modo de vida más tranquilo y en contacto con la naturaleza. Asimismo, como en los primeros dos casos anteriormente mencionados, la convivencia entre sectores sociales con capitales y modos de vida diferentes, llevó a la formación de identidades contrapuestas y a la formación de redes de sociabilidad diferenciadas. Por otra parte, el hecho de que la mayoría de estos nuevos habitantes llegaron desde centros suburbanos de la zona norte, nos indica que, como muestran algunas investigaciones acerca de la movilidad residencial en la RMBA, la elección de esta localidad como lugar de residencia estuvo también marcada por una búsqueda de cercanía respecto de sus barrios de origen.
Finalmente, el análisis de las experiencias urbanas de quienes trasladaron su residencia a la localidad de Ingeniero Maschwitz permite complejizar la imagen monolítica que suele darse al proceso de avance de la mancha urbana sobre las periferias. Los testimonios de los nuevos habitantes de la localidad aquí expuestos muestran la convivencia de elementos que los actores vinculan con "el campo", como el silencio, las arboledas y el barro, junto con la emergencia de problemas típicamente "urbanos" como los congestionamientos de autos y la contaminación sonora en avenidas y autopistas. Este caso muestra que, además de la difusión de enclaves de barrios cerrados y grandes centros comerciales comunicados por autopistas, algunos sectores de las periferias presentan también configuraciones barriales abiertas que atraen a individuos y familias de sectores medios urbanos y suburbanos. Sería interesante profundizar en el alcance de este fenómeno en las periferias de la ciudad a partir del estudio de otros casos análogos en el oeste y el sur de la RMBA, así como en las periferias de otros centros urbanos del país.

\section{Referencias}

Arizaga, C. (2017). Sociología de la felicidad. Autenticidad, bienestar y management del yo. Biblos.

Baer, L., Vecslir, L., \& Ciccolella, P. (2015). Buenos Aires, una metrópolis entre cambios e inercias tras la crisis. En M. territarios 45

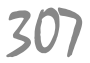


Schteingart \& P. Pirez (Coords.), Dos grandes metrópolis latinoamericanas: Ciudad de México y Buenos Aires (pp. 129-157). El colegio de México.

Ballent, A. (1998). Country Life: los nuevos paraísos, su historia y sus profetas. Block, (2), 88-101.

Ballent, A., \& Gorelik, A. (2001). País urbano y país rural: la modernización del país y su crisis. En A. Cattaruzza (Dir.), Crisis Económica, avance del estado e incertidumbre politica (19301943) (pp. 143-200). Sudamericana.

Benson, M., \& O'Reilly, K. (2009). Lifestyle migration: expectations, aspirations and experiences. Ashgate.

Bourdieu, P. (2000). Efectos de lugar. En P. Bourdieu, La Miseria del Mundo (pp. 119-124). Fondo de Cultura Económica.

Bozzano, H. (2007). Buenos Aires desde sus orígenes. Transformaciones territoriales y mutaciones productivas. En J. Borello (Coord.), Aproximaciones al mundo productivo de la Región Metropolitana de Buenos Aires (pp. 29-64). Universidad Nacional de San Martín.

Carmán, M. (2011). Las trampas de la naturaleza. Fondo de Cultura Económica.

Carmán, M., Vieira, N., \& Segura, R. (2013). Introducción. Antropología, diferencia y segregación urbana. En M. Carmán, N. Vieira, \& R. Segura (Coords.) Segregación y diferencia en la ciudad (pp. 11-35). FLACSO.
Carozzi, M. J. (2000). Nueva Era y terapias alternativas. Construyendo significados en el discurso y la interacción. UCA.

Ciccolella, P. (1999). Globalización y dualización en la Región Metropolitana de Buenos Aires. Grandes inversiones y reestructuración socioterritorial en los años noventa. EURE, 25(77), 5-27.

Ciccolella, P. (2009). Buenos Aires: una metrópolis postsocial en el contexto de la economía global. En P. Pírez (Ed.), Buenos Aires, la formación del presente (pp. 35-62). OLACCHI.

Ciccolella, P., Vecslir, L., \& Baer, L. (2015). Revitalización de subcentros metropolitanos. Buenos Aires entre la ciudad dispersa y la ciudad compacta. Contexto. 9(11), 11-27.

Cosacov, N. (2014). Trayectorias residenciales y decisiones de localización residencial de hogares de clase media residentes en el barrio de Caballito. Argumentos. Revista de critica social, (16), 41-70.

Cosacov, N. (2017). El papel de la familia en la inscripción territorial. Exploraciones a partir de un estudio de hogares de clase media en el barrio de Caballito, Buenos Aires. Población \& Sociedad, 24(1), 35-65. https://www.redalyc. org/articulo.oa? id=386951094002

Di Virgilio, M. (2014). Diferencias sociales en los procesos de movilidad residencial intraurbana en el Área Metropolitana de Buenos Aires (Argentina). Quivra, 16(1), 11-37. http://hdl. handle.net/11336/35531 
Funes, M. E. (2018). La espiritualización de lo cotidiano. Estilos de vida, experiencias espaciales y sectores medios en la periferia de Buenos Aires (Tesis de doctorado en Ciencias Sociales). Universidad de Buenos Aires; École des Hautes Études en Sciences Sociales.

Funes, M. E. (2021). Las sociabilidades situadas en el estudio de lo religioso. Análisis de una zona espiritual en la periferia de Buenos Aires. Religião e Sociedade, 4l(2).

Girola, M. F. (2004). Imaginarios urbanos en zonas verdes y zonas rojas de la Región Metropolitana de Buenos Aires. Cuadernos de Antropologia Social, (20), 93-111. https://doi. org/10.34096/cas.i20.4646

Gómez Pintus, A. (2013). Las formas de la expansión. 1910-1950. Barrios Parque $y$ loteos de fin de semana en la construcción del espacio metropolitano de Buenos Aires (Tesis doctoral en Arquitectura y Urbanismo). Facultad de Arquitectura y Urbanismo, Universidad Nacional de La Plata, La Plata.

González Bracco, M., \& Arizaga, C. (2019). Retóricas de lo auténtico: Estética y sensibilidad en los negocios gastronómicos de la Ciudad de Buenos Aires. En E. Licona Valencia \& A. Cortés Patiño (Coords.), Narrativas culinarias. Miradas etnográficas (pp. 102-118). Académica Española.

González, R., Otero, A., Nakayama, L., \& Marioni, S. (2009). Las movilidades del turismo y las migraciones de amenidad: Problemáticas y contradicciones en el desarrollo de centros turísticos de montaña. Revista de Geografía Norte Grande, (44), 75 92. https://doi.org/10.4067/S071834022009000300004

Gorelik, A. (2008). La aldea en la ciudad. Ecos urbanos de un debate antropológico. Revista del Museo de Antropologia, $1(1), 73-96$.

Gorelik, A. (2015). Terra incógnita. Para una comprensión del Gran Buenos Aires como Gran Buenos Aires. En G. Kessler (Dir.), Historia de la provincia de Buenos Aires. El Gran Buenos Aires (Tomo 6, pp. 21-71). Unipe; Edhasa. Guber, R. (2009). El salvaje metropolitano: Reconstrucción del conocimiento social en el trabajo de campo. Paidós.

Gutiérrez, A. (2007). De las redes de transporte al problema de la movilidad: límites físicos y analíticos de la expansión urbana en Buenos Aires. En J. Borello (Coord.), Aproximaciones al mundo productivo de la Región Metropolitana de Buenos Aires (pp. 239-266). Universidad Nacional de San Martín.

Gutman, M. (2007). Buenos Aires 15362006: historia urbana del Área Metropolitana. Infinito.

Heredia, M., \& Di Virgilio, M. (2012). Presentación. Dossier "Clase social y territorio". Quid, 16(2), 4-19. 
Instituto del Conurbano. (2014a). Densidad de población 2001 - Región Metropolitana de Buenos Aires. http:// observatorioconurbano.ungs.edu. ar/?page_id=2708

Instituto del Conurbano. (2014b). Densidad de población 2010 - Región Metropolitana de Buenos Aires. http:// observatorioconurbano.ungs.edu. ar/?page_id $=2708$

Instituto Nacional de Estadística y Censos (INDEC). (2001). Censo Nacional de Población Hogares y viviendas. https://www.indec.gob.ar/indec/ web/Nivel4-Tema-2-41-134

Instituto Nacional de Estadística y Censos (INDEC). (2010). Censo Nacional de Población Hogares y viviendas. https://www.indec.gob.ar/indec/ web/Nivel4-Tema-2-41-135

Instituto Nacional de Estadística y Censos (INDEC). (2020). Proyecciones por departamento. Cuadros estadisticos años 2010-2025. https://sitioanterior. indec.gob.ar/nivel4_default.asp?id_ tema_l=2\&id_tema_2 $=24 \&$ \&id_tema_3 $=119$

Lacarrieu, M., \& Girola, M. F. (2016). Modelos globales, procesos locales: Una mirada etnográfica sobre procesos de periurbanización en la Región Metropolitana de Buenos Aires. L'Ordinaire des Amériques, (207), 179-196. https://doi.org/10.4000/ orda. 3378

territarias 45

Magnani, J. G. C. (1999). Mystica urbe. Um estudo antropológico sobre o circuito neo-esotérico na metrópole. Studio Nobel.

Matarrita-Cascante, D. (2017). Moving the amenity migration literature forward: Understanding community-level factors associated with positive outcomes after amenity-driven change. Journal of Rural Studies, 53, 26-34. https://doi.org/10.1016/j. jrurstud.2017.05.004

Noel, G. (2011). Guardianes del paraíso. Génesis y genealogía de una identidad colectiva en Mar de las Pampas, Provincia de Buenos Aires. Revista del Museo de Antropologia, 4(1), 211-226. https:// doi.org/10.31048/1852.4826. v4.n1.5487

Noel, G. (2013). De los Códigos a los Repertorios: Algunos Atavismos Persistentes Acerca de la Cultura y una Propuesta de Reformulación. RELMECS, 3(2). http://www.memoria.fahce.unlp.edu.ar/art_revistas/ pr.6158/pr.6158.pdf

Noel, G. (2017). Ni lo uno ni lo otro, sino todo lo contrario: las limitaciones del dualismo rural-urbano en el abordaje de la región costera del Rio de la Plata y algunas propuestas de reconceptualización. Tessituras, 5(1), 129-170.

Noel, G., \& De Abrantes, L. (2014). La Gran División. Crecimiento y Diferenciación Social en una Ciudad Balnearia de la Costa Atlántica Bonaerene. Argumentos. Revista de crítica social, (16), 141-166. 
Perren, J., \& Cabezas, S. (2018). ¿Gentrificación en el "fin del mundo"? Crecimiento en altura y elitización en una ciudad intermedia de la Patagonia (Neuquén, 2001-2010). Quid, 16(9), 38-59.

Pírez, P. (2009). La privatización de la expansión metropolitana en Buenos Aires. En P. Pírez, (Ed.), Buenos Aires, la formación del presente (pp. 285304). OLACCHI.

Pizarro, C. (2010). Ruralidades emergentes en áreas periurbanas de los partidos de Escobar y Pilar. Revista Interdisciplinaria de Estudios Agrarios, 33, 87-127. http://hdl.handle. net/11336/112650

Prévôt-Schapira, M. -F. (2005). De l'usage de la fragmentation urbaine en Amérique latine. Bulletin de l'Association de Géographes Français, 82(4), 483495. https://doi.org/10.3406/bagf.2005.2481

Quirós, J. (2019). Nacidos, criados, llegados: Relaciones de clase y geometrías socioespaciales en la migración neorrural de la Argentina contemporánea. Revista Colombiana de Geografía, 28(2), 271-287. https://doi. org/10.15446/rcdg. v28n2.73512

Riera, V., Saccol, C., \& Wright, P. (2018). Cosmologías practicadas: un acercamiento estnográfico a la antroposofía. En P. Wright (Ed.), Periferias sagradas en la modernidad argentina (pp. 57-76). Biblos.
Ríos, D., \& y Pírez, P. (2008). Urbanizaciones cerradas en áreas inundables del municipio de Tigre: ¿́producción de espacio urbano de alta calidad ambiental? EURE, 34(101), 99-119. https://doi.org/10.4067/S025071612008000100005

Robert, F. (2000). Aproximación al tema de los barrios cerrados en la Región Metropolitana de Buenos Aires. Mundo Urbano, (1). http://www.mundourbano.unq.edu.ar/index.php/ ano-2000/37-numero-1-mayo/274-la-gran-muralla

Segura, R. (2012). Elementos para una crítica de la noción de segregación residencial socio-económica: Desigualdades, desplazamientos e interacciones en la periferia de La Plata. Quid, 16(2), 106-132. https://publicaciones.sociales.uba.ar/index.php/ quid 16/article/view/1118

Svampa, M. (2001). Los que ganaron. La vida en los countries y barrios privados. Biblos.

Torres, H. (1993). El mapa social de Buenos Aires (1940-1990). Dirección de Investigaciones, Secretaría de Investigación y Posgrado; FADU-UBA.

Torres, H. (2001). Cambios socioterritoriales en Buenos Aires durante la década de 1990. EURE, 27(80), 3556. https://doi.org/10.4067/S025071612001008000003

Torres, H. (2009). Procesos recientes de fragmentación socio-espacial en Buenos Aires: la suburbanización de territarias 45 
las élites. En P. Pírez (Ed.), Buenos Aires, la formación del presente (pp. 63-82). OLACCHI.

Trimano, L. (2017). Paisas y gringos. Neorruralidad serrana, transformaciones relacionales e identidades emergentes. Chungara Revista de Antropologia Chilena, 49(3), 461-471. https://doi.org/10.4067/S071773562017005000023.

Trimano, L. (2019). ¿¿Qué es la neorruralidad? Reflexiones sobre la construcción de un objeto multidimensional. Territorios, (41), 119-142. https:// doi.org/10.12804/revistas.urosario. edu.co/territorios/a.6951

Triví, N. (2018). Migración de amenidad, expansión urbana privatizada y turismo en el Valle del Conlara (San Luis, Argentina). Revista Huellas, 22(2), 11-33.

Viotti, N. (2017). Más allá de la terapia y la religión. Una aproximación relacional a la construcción espiritual del bienestar. Salud colectiva, 14(2), 241-256. Visacovsky, S. (2018). Clase media, un fenómeno multidimensional: Nuevos enfoques de investigación. Polis: observatorio de politicas públicas, 18, 28-32. http://hdl.handle.net/11336/93844 Williams, R. (2011). El campo y la ciudad. Paidós.

Wirth, L. (1945). Human ecology. American Journal of Sociology, 50(6), 483488. 